\title{
FRB microstructure revealed by the real-time detection of FRB170827
}

DOI:

10.1093/mnras/sty1122

\section{Document Version}

Accepted author manuscript

Link to publication record in Manchester Research Explorer

\section{Citation for published version (APA):}

Farah, W., Flynn, C., Bailes, M., Jameson, A., Bannister, K. W., Barr, E. D., Bateman, T., Bhandari, S., Caleb, M., Campbell-Wilson, D., Chang, S-W., Deller, A., Green, A. J., Hunstead, R. W., Jankowski, F., Keane, E., Macquart, J. P., Moller, A., Onken, C. A., ... Wolf, C. (2018). FRB microstructure revealed by the real-time detection of FRB170827. Monthly Notices of the Royal Astronomical Society, 478(1), 1209-1217. https://doi.org/10.1093/mnras/sty1122

\section{Published in:}

Monthly Notices of the Royal Astronomical Society

\section{Citing this paper}

Please note that where the full-text provided on Manchester Research Explorer is the Author Accepted Manuscript or Proof version this may differ from the final Published version. If citing, it is advised that you check and use the publisher's definitive version.

\section{General rights}

Copyright and moral rights for the publications made accessible in the Research Explorer are retained by the authors and/or other copyright owners and it is a condition of accessing publications that users recognise and abide by the legal requirements associated with these rights.

\section{Takedown policy}

If you believe that this document breaches copyright please refer to the University of Manchester's Takedown Procedures [http://man.ac.uk/04Y6Bo] or contact uml.scholarlycommunications@manchester.ac.uk providing relevant details, so we can investigate your claim.

\section{OPEN ACCESS}




\title{
FRB microstructure revealed by the real-time detection of FRB170827
}

W. Farah ${ }^{1}$, C. Flynn ${ }^{1,2}$, M. Bailes ${ }^{1}$, A. Jameson ${ }^{1,2}$, K. W. Bannister ${ }^{3}$, E. D. Barr ${ }^{4}$, T. Bateman ${ }^{5}$, S. Bhandari ${ }^{1,2}$, M. Caleb ${ }^{2,6,7}$, D. Campbell-Wilson ${ }^{5}$, S.-W. Chang ${ }^{2,6}$, A. Deller ${ }^{1,2}$, A. J. Green ${ }^{5}$, R. Hunstead ${ }^{5}$, F. Jankowski1 ${ }^{1,2,7}$, E. Keane ${ }^{2,8}$, J.-P. Macquart $^{3,9}$, A. Möller ${ }^{2,6}$, C. A. Onken ${ }^{2,6}$, S. Osłowski ${ }^{1}$, A. Parthasarathy ${ }^{1,2}$, V. Ravi $^{10}$, R. Shannon ${ }^{1,11}$, B. E. Tucker ${ }^{2,6}$, V. Venkatraman Krishnan ${ }^{1,2}$, C. Wolf ${ }^{2,6}$

\footnotetext{
${ }^{1}$ Centre for Astrophysics and Supercomputing, Swinburne University of Technology, Mail H30, PO Box 218, VIC 3122, Australia

${ }^{2}$ ARC Centre of Excellence for All-sky Astrophysics (CAASTRO)

${ }^{3}$ ATNF, CSIRO Astronomy and Space Science, PO Box 76, Epping, NSW 1710, Australia

${ }^{4}$ Max-Plank-Institute für Radioastronomie, Auf dem Hügel 69, D-53121 Bonn, Germany

${ }^{5}$ Sydney Institute for Astronomy, School of Physics A28, University of Sydney, NSW 2006, Australia

${ }^{6}$ Research School of Astronomy and Astrophysics, Australian National University, Canberra, ACT 2611, Australia

${ }^{7}$ Jodrell Bank Centre for Astrophysics, School of Physics and Astronomy, The University of Manchester, Manchester M13 9PL, UK

${ }^{8}$ SKA Organization, Jodrell Bank Observatory, Cheshire SK11 9DL, UK

${ }^{9}$ International Centre for Radio Astronomy Research, Curtin University, Bentley, WA 6102, Australia

${ }^{10}$ Cahill Centre for Astronomy and Astrophysics, MC 249-17, California Institute of Technology, Pasadena, CA 91125, USA

${ }^{11}$ The Australian Research Council Centre of Excellence for Gravitational Wave Discovery (OzGrav)
}

16 March 2018

\begin{abstract}
We report a new Fast Radio Burst (FRB) discovered in real-time as part of the UTMOST project at the Molonglo Observatory Synthesis Radio Telescope (MOST). FRB170827 is the first detected with our low-latency $(<24 \mathrm{~s})$, machine-learning-based FRB detection system. The FRB discovery was accompanied by the capture of voltage data at the native time and frequency resolution of the observing system, enabling coherent dedispersion and detailed off-line analysis, which have unveiled fine temporal and frequency structure. The dispersion measure (DM) of $176.80 \pm 0.04 \mathrm{pc} \mathrm{cm}^{-3}$, is the lowest of the FRB population. The Milky Way contribution along the line of sight is $\sim 40 \mathrm{pc} \mathrm{cm}^{-3}$, leaving an excess DM of $\sim 145 \mathrm{pc} \mathrm{cm}^{-3}$. The FRB has a fluence $>$ $20 \pm 7$ Jyms. The burst's width at $10 \%$ maximum is $400 \mu \mathrm{s}$, making it one of the narrowest FRBs. The burst shows three temporal components, the narrowest of which is $\sim 30 \mu \mathrm{s}$, and a scattering of $4.1 \pm 2.7 \mu \mathrm{s}$. The FRB shows spectral modulations on frequency scales of $1.5 \mathrm{MHz}$ and $0.1 \mathrm{MHz}$. Both are prominent in the dynamic spectrum, which shows a bright region of emission between 841 and $843 \mathrm{MHz}$, and weaker, patchy emission across the entire band. We show the fine spectral structure could arise in the FRB host galaxy, or its immediate vicinity.
\end{abstract}

\section{INTRODUCTION}

Fast Radio Bursts (FRBs) form a class of extragalactic radio transients, with approximately 30 published since Lorimer et al. (2007) reported the first. The dispersion measures (DMs) of known FRBs currently spans the range $175-2600$ pc $\mathrm{cm}^{-3}$ (FRBcat; Petroff et al. 2016), vastly exceeding the contribution of the Milky Way along their line of sight. FRBs have been detected at the Green Bank Telescope (GBT), the Parkes radio telescope, the Arecibo Observatory, the upgraded Molonglo Observatory Synthesis Telescope (UTMOST) and the Australian Square Kilometer Array Pathfinder (ASKAP) (Lorimer et al. 2007, Keane et al. 2012, Burke-Spolaor \& Bannister 2014; Spitler et al. 2014.
Petroff et al. |2015; Ravi et al. 2015; Champion et al. 2016. Masui et al. 2015 Keane et al. 2016 Ravi et al. 2016 | Petroff et al. 2017, Caleb et al. 2016; Bannister et al.|2017, Bhandari et al. 2018)

Bhandari et al. (2018) have recently estimated the FRB event rate from FRBs found in the HTRU (Keith et al. 2010) and SUPERB (Keane et al. 2018) surveys at Parkes as $1.7_{-0.9}^{+1.5} \times 10^{3} \mathrm{FRBs}(4 \pi \mathrm{sr})^{-1}$ day $^{-1}$ above $\sim 2$ Jy ms. At 19 FRBs, this is the largest sample of FRBs found with a single instrument. The authors show that there is no strong evidence that FRBs are other than isotropically distributed on the sky, although the sample size remains small. Macquart \& Ekers (2018) found that the cumulative source count distribution of Parkes FRBs is rather steep, implying a non-

(C) 0000 The Authors 
uniform space density with distance or source population evolution. Larger samples are required to address this issue conclusively, and are expected to become available as facilities like ASKAP (Bannister et al. 2017) and CHIME (Bandura 2014) reach full FRB search capacity.

Only one of the FRBs reported to date has been found to repeat (FRB121102; Spitler et al.|2016), with the emission showing no sign of an underlying periodicity. The opportunity for repeated targeting of this source has led to a precise localisation using radio interferometers, with FRB121102 pinpointed to a low-metallicity dwarf galaxy at a redshift of $z=0.193$ (Chatterjee et al. 2017, Tendulkar et al. 2017 , Bassa et al. 2017), co-located to within a region $\lesssim 12$ mas with a persistent radio source (Marcote et al. 2017). The properties of the host galaxy of FRB121102 are similar to those of the hosts of hydrogen-poor superluminous supernovae (SLSNe-I), leading Metzger et al. (2017) to propose that the repeated bursts from FRB121102 originate from a young magnetar remnant embedded within a young hydrogen-poor supernova remnant which would be at most a few decades old. Michilli et al. (2018) have recently shown that the bursts of FRB121102 exhibit extreme Faraday rotation measures, implying that the source resides in a highly magnetised region.

Many theories have been formulated to explain FRBs, and can be broadly classified as non-cataclysmic and cataclysmic, depending on the fate of the progenitor. Noncataclysmic theories include giant flares from magnetars (Pen \& Connor 2015), compact objects in young supernovae (Metzger et al. 2017) and supergiant pulses from extragalactic neutron stars (Cordes \& Wasserman 2016). Cataclysmic events include scenarios such as neutron star mergers (Totani 2013) and "blitzars" which occur when a spinning-down neutron star collapses into a black hole (Falcke \& Rezzolla 2014), releasing the neutron star's magnetosphere. Cataclysmic models for the events are challenged by the existence of the repeating FRB, although it is not clear whether FRB121102 is typical of the entire FRB population (Palaniswamy et al. 2018).

A key element to a better understanding of FRBs is to localise them to their host galaxies. We are currently limited to $\approx 10$ arcmin radius localisations of FRBs at Parkes, GBT and ASKAP, or narrow fan-beam localisations with UTMOST which are $5 \operatorname{arcsec} \times 1.2$ degrees. Plans are currently afoot at UTMOST (the UTMOST-2D project), ASKAP (Bannister et al. 2017), MeerKAT, and the Very Large Array (Law et al. 2015), to achieve localisations ranging from a few square arcminutes to a square arcsecond or less. The North-South arm of the Molonglo telescope, which crosses at right angles relative to the East-West, is currently being fitted out to approximately the same sensitivity (fluence $\approx 5 \mathrm{Jy} \mathrm{ms}$ for a $1 \mathrm{~ms}$ burst) as the East-West arm. The localisation that these facilities plan to deliver in the coming 24 months is in the range of $\approx 10 \operatorname{arcsec}$ down to subarcsecond scales, enabling host galaxy identifications for FRBs (Eftekhari \& Berger 2017).

Contingent to source localisation at UTMOST is the capacity to do real-time detection of FRBs. Due to the RFI (Radio Frequency Interference) environment at the site, it is advantageous to use a machine-learning-based system to avoid false triggers so that voltage data can confidently be recorded when FRB events occur. We have developed such
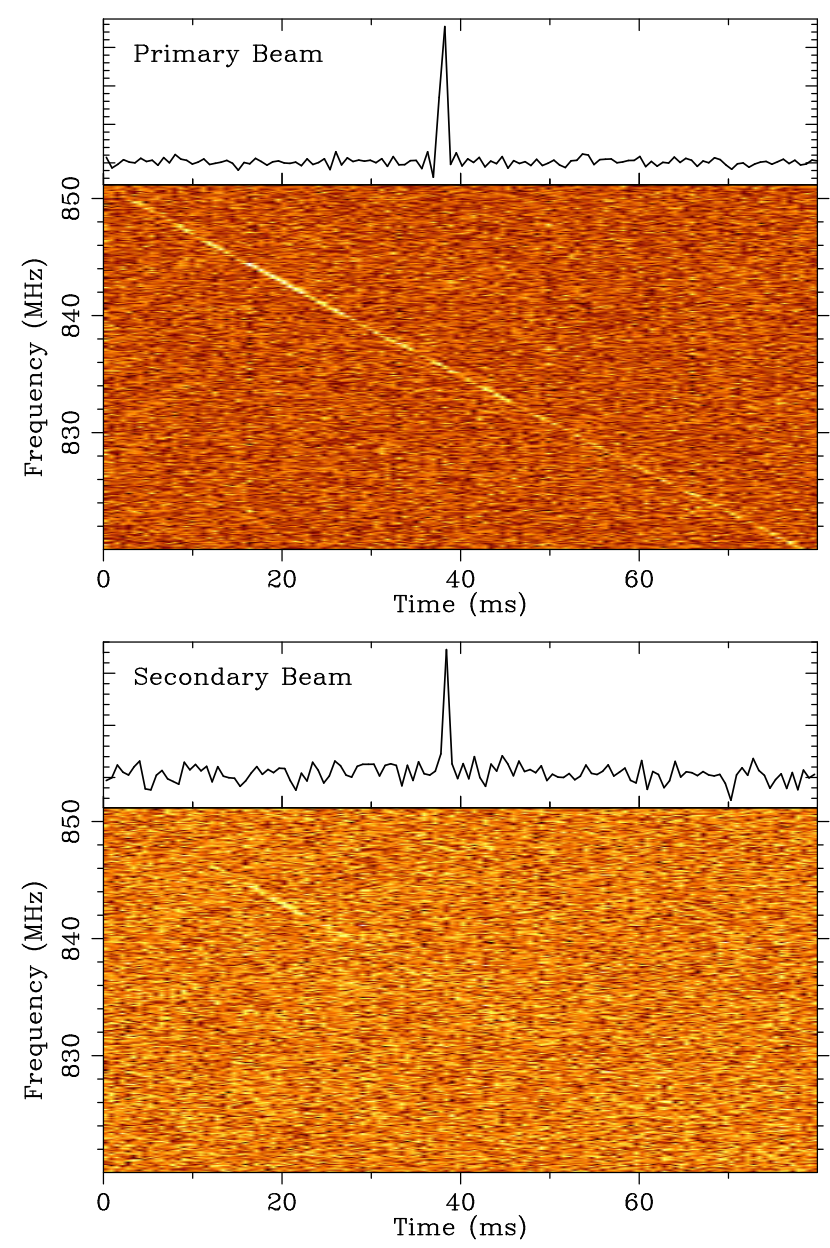

Figure 1. Top: Fan-beam of primary detection of FRB170827 at a resolution of $327.68 \mu$ s and $97.66 \mathrm{kHz}$. Bottom: adjacent fanbeam detection. The detection of FRB170827 in only two fanbeams is consistent with a source originating in the far field.

a system, and the FRB reported here is our first detection since the system sensitivity was improved substantially by hardware changes implemented in July 2017. Voltage capture not only leads to superior localisation of the FRB, but it also gives remarkable temporal and frequency resolution relative to our now superseded system.

In this paper we report the first FRB for which a voltage capture has been achieved after a real-time discovery. In section 2, we describe Molonglo's observing setup, and the real-time pipeline. We report the discovery of FRB170827 in section 3. The analysis of the dynamic spectrum and pulse profile of the FRB is presented in section 4 In section 5 , we present the conducted multiwavelength followup. We discuss our results in section 6

\section{OBSERVATIONS AND THE UTMOST DATA CAPTURE SYSTEM}

The UTMOST is an on-going project to transform MOST, a radio interferometer located near Canberra, Australia, into an FRB finder (Bailes et al. 2017). The $1.6 \mathrm{~km}$ extent of UTMOST reflector is divided into 352 "modules", where each module consists of 22 ring antennas that select a single 
right circular polarisation and allow the module beam to be steered. The central observing frequency of Molonglo is 835 $\mathrm{MHz}$ and the total bandwidth is $31.25 \mathrm{MHz}$. Since 2017 July, we have been operating the UTMOST telescope as a transit facility, and steering of the array away from the meridian has been sacrificed for better sensitivity. The mechanical system responsible for phasing these antennas away from the meridian was approaching end-of-life and had become unreliable when slewing, such that the sensitivity had fallen well below that in early 2016 when three FRBs were discovered (Caleb et al. 2016). With the switch to transit mode the telescope sensitivity improved approximately three-fold. The system is proving to be very stable, with phasing calibration of the system required only a few times weekly.

\subsection{Real-time analysis and voltage capture}

The real-time FRB analysis system, at its core, comprises a GPU-based single-pulse processing software, HEIMDALL The input to HEIMDALL is the "HIRES" filterbank data $(327.68 \mu \mathrm{s}, 97.66 \mathrm{kHz}, 8 \mathrm{bit})$ for each of the 352 fan-beams: equally spaced tied array beams that cover the field of view of an individual UTMOST module. HEIMDALL searches the fan-beam data streams, spread across 8 GPUs. The software performs dedispersion (from 0 to $2000 \mathrm{pc} \mathrm{cm}^{-3}$ ) and matched filtering (from $327.68 \mu$ s to $83.886 \mathrm{~ms}$ ), and reports time-stamped FRB candidates. Since part of the UTMOST operating spectrum is allocated to mobile phone communications, the vast majority of FRB candidates are artifacts, numbering tens of thousands per night. The system was thus extended by a supervised machine-learning algorithm, based on random forest (Breiman 2001), in order to perform real-time candidate classification. The model was trained on single pulses from various pulsars, and on RFIgenerated candidates, achieving an accuracy of $98.8 \%$ (10fold cross-validation). To perform classification, the model is specified by predefined features, extracted from the input data stream of a given candidate. These features are DM and $\mathrm{S} / \mathrm{N}$ agnostic, and sufficient to characterise the RFI activity during an event, the noise statistics, and the validity of the candidate. The details of the pipeline will be presented in a subsequent paper.

Application of the pipeline reduced the number of candidates to a manageable number, $\sim 10$ a day, the first step to making voltage capture a possibility. Channelised voltages are buffered in Random Access Memory for 24 seconds, sufficient to perform real-time beam-forming, single pulse searching and classification. Upon a trigger, a voltage capture around an interesting event is performed, while accounting for dispersive delay and allowing a narrow buffer window for baseline estimation. The captured data are the channelised, critically sampled voltages from each antenna that have not been subject to any RFI mitigation. An email is then issued to human inspectors for a final assessment of the candidate.
Table 1. Properties of FRB170827

\begin{tabular}{lc} 
Event UTC & $2017-08-2716: 20: 18.1$ \\
Fan-beam number & 92 \\
S/N (detection fan-beam) & 48 \\
S/N (coherently dedispersed) & 110 \\
Sampling time & $327.68 \mu \mathrm{s}$ \\
Detection Filter & $(655.36 \mu \mathrm{s}$ width) \\
Best-fitting $\alpha$ (h:m:s) & $00: 49: 18.66(\mathrm{~J} 2000)$ \\
Best-fitting $\delta$ (d:m:s) & $-65: 33: 02.5(\mathrm{~J} 2000)$ \\
Galactic longitude $l$ & $303.29^{\circ}$ \\
Galactic latitude $b$ & $-51.58^{\circ}$ \\
$S_{\text {peak }}$ (lower limit) & $60 \pm 20 \mathrm{Jy}^{*}$ \\
Observed Fluence (lower limit)* & $20 \pm 7 \mathrm{Jy} \mathrm{ms}^{*}$ \\
Width (at 10\% maximum) & $400 \pm 10 \mu \mathrm{s}$ \\
Refined DM & $176.80 \pm 0.04 \mathrm{pc} \mathrm{cm}^{-3}$ \\
Galactic DM (NE2001) & $37 \mathrm{pc} \mathrm{cm}^{-3}$ \\
Galactic DM (YMW16) & $26 \mathrm{pc} \mathrm{cm}^{-3}$ \\
\hline * Corrected for the known position of the FRB within the \\
primary beam pattern in the East-West direction, but \\
uncorrected for the (unknown) FRB position in the north- \\
south direction.
\end{tabular}

\section{DISCOVERY OF FRB170827}

Fig. 1 shows the dynamic spectrum for the FRB at its detection resolutions of $327.68 \mu \mathrm{s}$ and $97.66 \mathrm{kHz}$. After correcting for the effect of interstellar dispersion, and averaging across the frequency axis, the event $\mathrm{S} / \mathrm{N}$ at this time resolution was 48 . Within seconds, it was evaluated as an FRB candidate by our machine-learning system, triggering an email alert and a voltage dump of $\sim 270 \mathrm{~ms}$ around the event. A failure of the plotting routine (ironically due to the very high $\mathrm{S} / \mathrm{N}$ of the event, and since corrected) meant that FRB170827 was only identified as a bona fide FRB three days after the event, which limited immediate follow-up at other wavelengths. The result was issued as Astronomer's Telegram ATel 10697 (Farah et al. 2017).

The event was seen in 2 fan-beams only (Fig. 1), consistent with celestial sources and the spacing of our fan-beams on the sky. We searched all other fan-beams for similar events in time and dispersion measure, finding none above a $\mathrm{S} / \mathrm{N}$ of 8 . The voltage data were particularly clean around the time of the event, as expected in the very early morning hours (local time $2 \mathrm{AM}$ ) on site, as RFI locally is dominated by mobile handset traffic.

Molonglo's backend supports a mode in which saved voltages can be read and processed from disk, rather than live from the telescope. Voltages allow us to make an improved localisation of the FRB, as we can place fan-beams on the sky arbitrarily. We placed 352 fan-beams across 0.04 deg (beam spacing of $1.1396 \times 10^{-4}$ degrees) covering the two detection fan-beams, compute the $\mathrm{S} / \mathrm{N}$ of the FRB in each, and fit for the sky position which maximises $\mathrm{S} / \mathrm{N}$. The best-fitting central position for the FRB is $\alpha=00: 49: 17.68$, $\delta=-65: 33: 02.5$ (J2000). Our localisation region is of order 5 arcsec (1-sigma) in the east-west direction, but is constrained only by the telescope primary beam in the northsouth direction, with 1-sigma localisation error of 1.2 degrees. The localisation arc can be described as:

$$
\begin{array}{r}
\delta=-60.71088-253.7786 \times(\alpha-0.8) \\
+1480.220 \times(\alpha-0.8)^{2},
\end{array}
$$

1 https://sourceforge.net/projects/heimdall-astro/ 


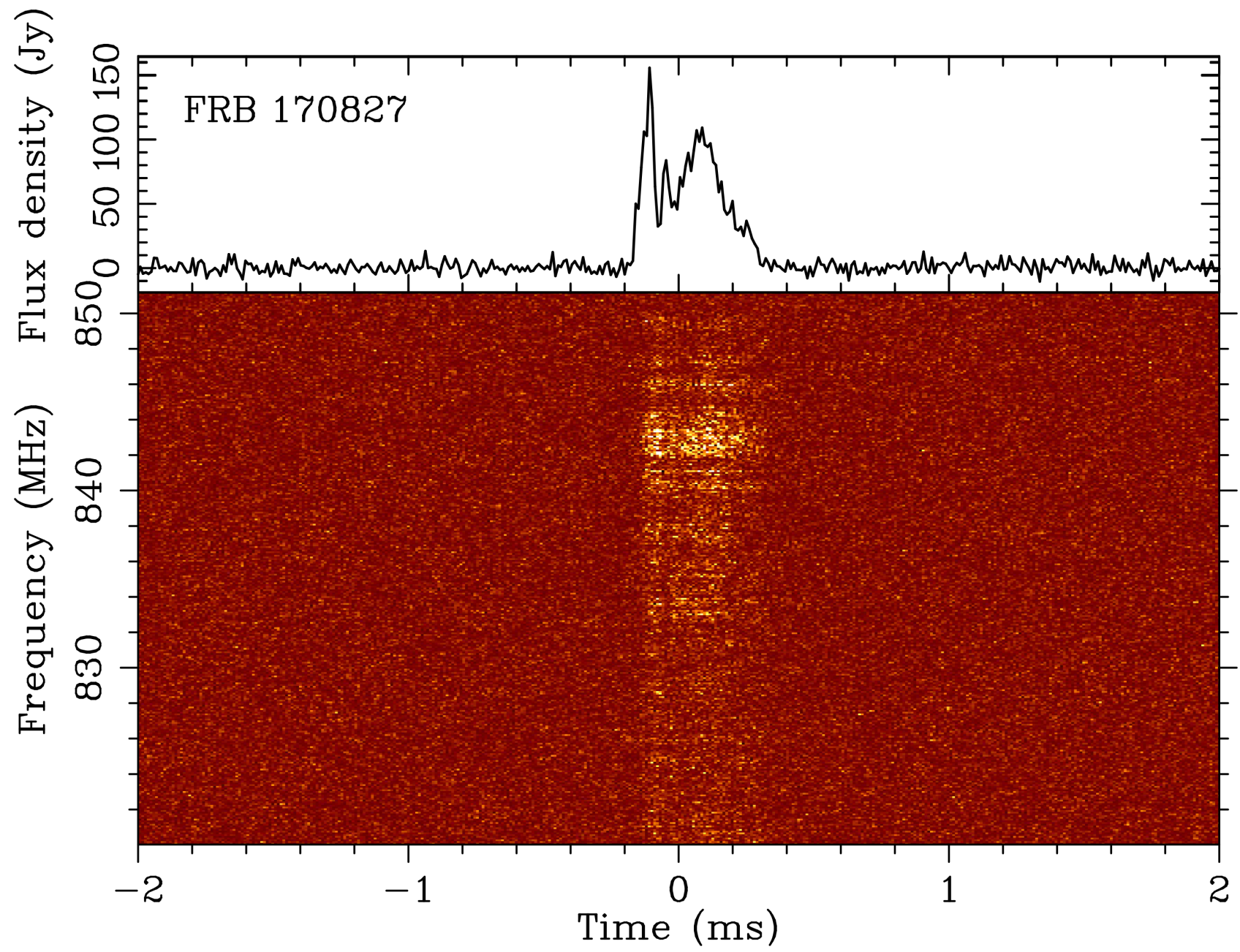

Figure 2. FRB170827 coherently dedispersed with dispersion measure $=176.8 \mathrm{pc}^{-3}$. Discernible structure in FRB170827 is seen at the highest available resolution of the instrument at $10.24 \mu \mathrm{s}$ and $97.66 \mathrm{kHz}$.

where $\alpha$ is in hours, $\delta$ is in deg, and is valid in the $\alpha$ range $[0.81,0.84]$. Once the position was optimized, the voltages were streamed through the system again to form a tiedarray beam on the target position. The signal was coherently dedispersed over a range of DMs, including a correction for the system gain as a function of frequency (using weights previously obtained from the bright southern pulsar J1644-4559), to determine the DM that maximizes the S/N ratio of the event. We obtain a DM of $176.80 \pm 0.04 \mathrm{pc} \mathrm{cm}^{-3}$ and an $\mathrm{S} / \mathrm{N}$ of 110 (compared to $\mathrm{S} / \mathrm{N}=48$ obtained in the detection fan-beam).

The event was sufficiently bright in the detection fanbeam to saturate $\sim 10 \%$ of the 8 bit, high-resolution filterbank data samples (resolution $327.68 \mu \mathrm{s}, 97.66 \mathrm{kHz}$ ) in a $\sim 1 \mathrm{~ms}$ window centered on the event. This is the primary reason for the substantial enhancement in the $\mathrm{S} / \mathrm{N}$ from 48 to 110: the much higher dynamic range in the voltage data allows us to fully recover the lost flux density. This loss of signal is expected as the parameters used to scale the data stream down to 8-bits are optimised to maximise our sensitivity to $\sim 10 \mathrm{~S} / \mathrm{N}$ events, while not limiting our ability to discover bright events. The $\mathrm{S} / \mathrm{N}$ improvement is also, to a lesser extent, due to the use of coherent dedispersion, lo- calisation of the event within the primary beam, solving for an optimal DM and improved time resolution of this narrow event.

Fig. 2 shows the event at resolutions of $10.24 \mu$ s and $97.66 \mathrm{kHz}$, after coherent dedispersion and bandpass correction.

The DM estimate for the Galaxy contribution along the FRB's line of sight is $37 \mathrm{pc} \mathrm{cm}^{-3}$ using the NE2001 model and $26 \mathrm{pc} \mathrm{cm}^{-3}$ using the YMW16 model. This results in an average DM excess of $\sim 145 \mathrm{pc} \mathrm{cm}^{-3}$ for the FRB, the lowest value for any FRB published. The upper limit on the DM-inferred redshift is thus $\lesssim 0.12$ (Inoue 2004). The observational properties of FRB170827 are listed in Table 1.

\subsection{Possible SMC Origin?}

The extension of the localisation arc given by Eq. 1 southward by 7 degrees (6-sigma) intersects with the central regions of the Small Magellanic Cloud (SMC). The DM of the event is similar to the DM of pulsars in the central regions of the SMC, leading us to question whether the source could be in the SMC and we have seen a bright event in a sidelobe. In Fig. 3 we show the DM distribution as a function of 
Galactic latitude of FRBs published to date, compared with pulsars in the Milky Way, and in the Small and Large Magellanic Clouds respectively, showing that FRB170827 overlaps in DM with pulsars in the SMC. Tests of the scenario that FRB170827 is a far sidelobe detection were carried out in the week following the event, in which the telescope was moved 7 degrees south of the bright southern pulsar Vela, searching for single pulse events. We found that 7 degrees off boresight to the south of Vela, occasional pulses could be seen from Vela at a $\mathrm{S} / \mathrm{N}$ of a few percent of the boresight $\mathrm{S} / \mathrm{N}$. We estimate that if the source were actually in the SMC, it could be detected at boresight with a $\mathrm{S} / \mathrm{N} \gtrsim 1000$, and would have a peak flux density of $\gtrsim 2 \mathrm{kJy}$. We compare such a source to the Crab pulsar in the Milky Way as follows: 2 $\mathrm{kJy}$ pulses at the distance of the SMC would correspond to $\sim 2$ MJy pulses at the distance of the Crab. The brightest pulses from the Crab, seen in of order $1 \mathrm{hr}$ of observation, are $150 \mathrm{kJy}$ at $0.4 \mathrm{GHz}$ or $4 \mathrm{kJy}$ at $1.4 \mathrm{GHz}$, and $\sim$ a few $100 \mu$ s wide (Cordes et al. 2004). FRB170827 would need to be $\sim 50$ times brighter than the most luminous giant pulses emitted by the Crab. It is likely that a source capable of such pulses in the SMC, if Crab-like, would have given off large numbers of fainter pulses that should have been seen in extant SMC surveys (e.g. Manchester et al. 2006). We therefore consider it unlikely that FRB 170827 resides in the SMC. Pulsar wind nebulae were also searched for along the localisation arc, and none were found ${ }^{2}$.

\section{ANALYSIS OF FRB170827}

Voltage data were captured for $270 \mathrm{~ms}$ encompassing the event, and allowed us to examine the FRB's temporal and frequency structure with much higher resolution than for all of the non-repeating published FRBs. The dynamic spectrum of the FRB displayed in Fig. 2 shows a region of enhanced emission between 841 and $843 \mathrm{MHz}$, striations on a scale of 100 to $200 \mathrm{kHz}$ and "spiky" emission features which can be as bright as $1 \mathrm{kJy}$. Interestingly, the latter two features are similar to what is seen in FRB150807 (Ravi et al. 2016).

\subsection{Spectral Modulation}

Point-like radio sources scintillate due to propagation through inhomogeneous dispersive media. To measure the scintillation effects, we construct the frequency autocovariance function (ACF) of the spectrum $S(\nu)$ :

$$
A(\Delta \nu)=\frac{1}{N} \sum_{\nu} \Delta S(\nu) \Delta S(\nu+\Delta \nu),
$$

where $\Delta S(\nu)=S(\nu)-\bar{S}$, with $\bar{S}$ being the mean flux density, and $N$ the number of frequency channels. The zero lag

${ }^{2}$ Roberts, M.S.E., 2004, 'The Pulsar Wind Nebula Catalog (March 2005 version)', McGill University, Montreal, Quebec, Canada (available on the World-Wide-Web at http://www. physics.mcgill.ca/ pulsar/pwncat.html).

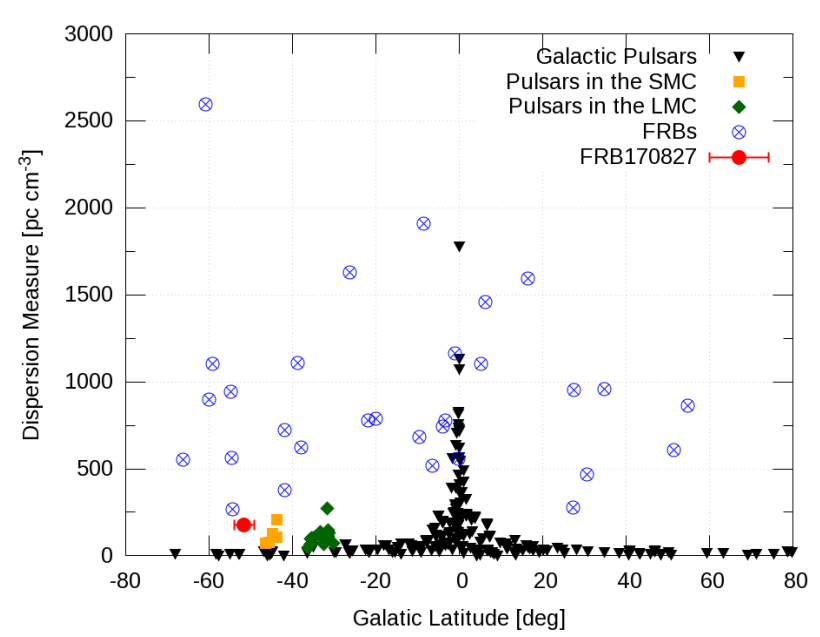

Figure 3. DM versus Galactic latitude plot for published FRBs and Milky Way, LMC and SMC pulsars. Galactic pulsars are shown in black, while pulsars in the LMC and SMC are shown in orange and green respectively. FRBs available on the FRBcat are displayed in blue. FRB170827 is shown in red and has a DM which is similar to pulsars in the $\mathrm{SMC}$, and a sky position $\approx 7$ degrees directly north of the SMC, such that the extension of the localisation arc for the FRB source passes through the SMC centre. Nevertheless, we argue in section 3.1 that it is unlikely that the FRB was a giant pulse emitted by a pulsar in the SMC that was detected in a far sidelobe.

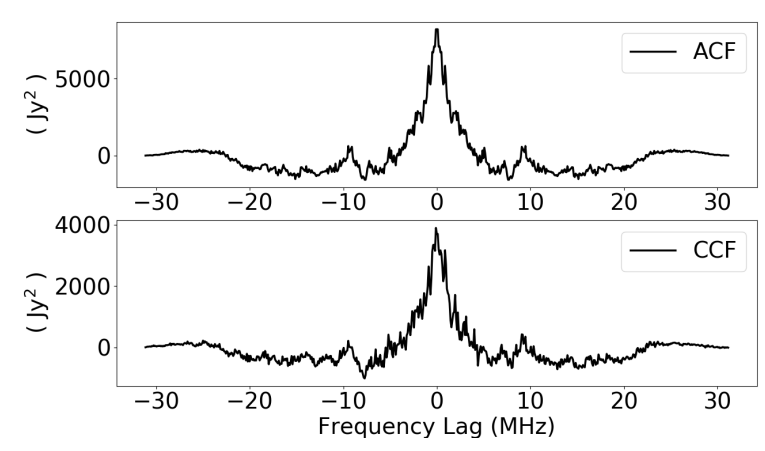

Figure 4. Auto-covariance function (top) of the spectrum of FRB170827, and the cross-covariance function (bottom) of the leading and trailing spectra of the FRB.

value, associated with self noise, was excised from the autocovariance function. The ACF was then normalised by its maximum and fitted by a Gaussian function of the form:

$$
\xi(\Delta \nu)=\exp \left[-\mathrm{b} \Delta \nu^{2}\right]
$$

The constructed ACF is shown in Fig. 4. The decorrelation bandwidth, $\Delta \nu_{\mathrm{d}}$, is usually defined as the frequency lag where the ACF decays to half power (Cordes 1986). The computed $\Delta \nu_{\mathrm{d}} \sim 1.5 \mathrm{MHz}$ is consistent with what is expected along the line of sight, as shown by the NE2001 model (Cordes \& Lazio 2002). In Fig. 4, we show the cross- 
covariance function $(\mathrm{CCF})$ of the spectra of the leading and trailing features of the temporal profile (see $\S 4.2$. The CCF peaks at zero-lag, and shows only modest asymmetry that is consistent with arising from sample variance and noise. If the scintillation patterns are identical between the two feature windows, the ACF and $\mathrm{CCF}$ would have the same shape (Cordes et al. 1983). We conclude that the dynamic spectrum of FRB170827 is consistent with arising from scintillation.

Another notable feature of the dynamic spectrum of FRB170827 is the $100-200 \mathrm{kHz}$ wide striations seen across the pulse profile. These cannot be explained by a passage through the ISM, as they are an order of magnitude narrower than is expected for the line of sight for the NE2001 model $(1.5 \mathrm{MHz})$. Below, we consider the possibility that they arise in a second scattering screen, well outside the Milky Way.

\subsection{Profile Temporal Structure}

Apart from the repeating FRB, no other FRB has been studied at this timescale due to limitations set by DM smearing (e.g. Bhandari et al. 2018). Although FRB170827 is much narrower than most FRBs, this might just be an observational bias (Ravi 2017).

The frequency-averaged pulse profile of the burst shows temporal modulation of the order of tens of microseconds and the profile can be divided into three different components: a sharp leading feature with a peak flux density $\gtrsim 100 \mathrm{Jy}$ and width $\sim 50 \mu \mathrm{s}$, an intermediate feature, and a trailing feature. While most FRBs appear to have a single temporal component, some have shown multiple peaks (e.g. FRB121002; Champion et al. 2016). The pulse profile was fitted with a model $\mathbb{G}$ described by the summation of three Gaussian profiles, each convolved with a one-sided exponential, of the form:

$$
\begin{aligned}
\mathbb{G}_{i}\left(t \mid \mathrm{A}_{i}, \Delta t_{i}, \sigma_{i}, \tau\right) & =\mathrm{A}_{i} \times\left[\exp \left(-\frac{\left(t-\Delta t_{i}\right)^{2}}{2 \sigma_{i}^{2}}\right)\right] \\
* & {\left[\mathbb{H}\left(t-\Delta t_{i}\right) \exp \left(-\frac{t-\Delta t_{i}}{\tau}\right)\right], }
\end{aligned}
$$

$$
\text { and } \mathbb{G}=\mathbb{G}_{1}+\mathbb{G}_{2}+\mathbb{G}_{3},
$$

where $t$ is time in microseconds, $\tau$ is the scattering timescale at $835 \mathrm{MHz}, \mathbb{H}(t)$ is a Heaviside unit step function, $\Delta t$ is the time offset of a Gaussian relative to the first, $\sigma$ is the Gaussian dispersion width, and the asterisk denotes convolution. The optimal parameters of the model were obtained using Markov chains Monte Carlo (MCMC) ${ }^{3}$ Fig. 5 shows the best-fitting model $\mathbb{G}$ overlaid on the data, and the optimal model parameters are presented in Table 2 . We show the estimated posterior distributions for the model parameters in Fig. $6^{4}$

We estimate the scattering timescale $\tau$ to be $4.1 \pm 2.7$ $\mu \mathrm{s}$. This corresponds to frequency structures on a scale of

3 Using the PYTHON package EMCEE (Foreman-Mackey et al. 2013)

4 'This figure is made using the public python package CORNER (Foreman-Mackey 2016). $\sim(2 \pi \tau)^{-1}$, consistent with the striations on the 100-200 $\mathrm{kHz}$ scale seen in the dynamic spectrum.

In the following sub-section, we assume that this fine structure is induced by a second scattering screen, external to the Milky Way. We note that $\tau$ is poorly constrained on the lower end of the posterior distribution, and the data are consistent with no scattering at the 2-sigma level. In this case, the structure may be intrinsic to the emission process of the source; this possibility is beyond the scope of the present paper.

\subsection{Two Screen Model}

The dynamic spectrum of FRB170827 shows scintillation on two frequency scales: broadband features explained by a passage through the turbulent ISM, and a finer structure with striations 1-2 frequency channels $(100-200 \mathrm{kHz})$ wide, hinting at the presence of another scattering screen along the FRB's path from the host to the observer. In this section, we assume that both spectral modulation scales arise from scintillation induced by two scattering screens, a Galactic screen, and another closer to source.

We model the near screen to be placed $\sim 1 \mathrm{kpc}$ from the observer, with a broadening timescale of $\tau_{\text {ISM }}=$ $\left(2 \pi \Delta \nu_{\mathrm{d}, \mathrm{ISM}}\right)^{-1} \approx 0.1 \mu \mathrm{s}$. The second screen, in order to give rise to the fine spectral modulation, should be sufficiently distant that it is not resolved by the Galactic screen. Scintillations from a far screen will only be apparent if the incident wave field is spatially coherent across the transverse extent of the scattering disk of the near screen. This scale is of order the screen's refractive scale, $r_{\text {ref }}$, defined as:

$$
r_{\mathrm{ref}}=\theta_{\mathrm{d}} \times D=\frac{r_{\mathrm{F}}^{2}}{s_{0}},
$$

where $\theta_{\mathrm{d}}$ is the angular radius of the source's broadened image, $D$ is the distance to the scattering screen, $r_{\mathrm{F}}$ is the Fresnel scale, and $s_{0}$ is the field coherence scale (Lorimer \& Kramer 2012).

We can obtain a first order estimate of the coherence scale of the much further screen by estimating the scale on which its average visibility declines. For a source at an angular diameter distance $D_{\mathrm{S}}$ from the observer and an angular diameter distance $D_{\text {LS }}$ from the (more distant) lensing screen, this scale is:

$$
r_{0}=s_{0, \mathrm{FAR}} \times \frac{D_{\mathrm{S}}}{D_{\mathrm{LS}}},
$$

(e.g. Macquart \& Koay 2013). The scintillation bandwidth $\Delta \nu_{\mathrm{d}}$ is related to the coherence scale of the scattering screen $s_{0}$ by:

$$
\sqrt{\frac{\Delta \nu_{\mathrm{d}}}{\nu}}=\frac{s_{0}}{r_{\mathrm{F}}}
$$

(Lorimer \& Kramer 2012). For the Galactic screen, we have:

$$
r_{\mathrm{F}, \mathrm{ISM}}=\sqrt{\frac{D_{\mathrm{ISM}}}{k}},
$$

where $k$ is the radiation angular wavenumber. Taking into account the curved geometry of spacetime, the coherence 
Table 2. Best-fitting parameters to the FRB170827 pulse profile, using 3 Gaussian components with exponential scattering tails (see Eq. 4. $\mathbb{G}_{1}, \mathbb{G}_{3}, \mathbb{G}_{3}$ represent the leading, intermediate and trailing features of the FRB170827 pulse profile. The parameter $\Delta t$ is the time offset of the feature relative to the leading feature, $\sigma$ is the Gaussian dispersion width of each feature. and $\tau$ is the scattering timescale.

\begin{tabular}{cccc}
\hline & $\Delta t(\mu \mathrm{s})$ & $\sigma(\mu \mathrm{s})$ & $\tau(\mu \mathrm{s})$ \\
\hline $\mathbb{G}_{1}$ & $0.0_{-0.7}^{+0.7}$ & $22.1_{-0.8}^{+0.8}$ & \\
$\mathbb{G}_{2}$ & $66.2_{-1.5}^{+1.3}$ & $7.5_{-2.0}^{+1.2}$ & $4.1_{-2.7}^{+2.7}$ \\
$\mathbb{G}_{3}$ & $199.0_{-2.0}^{+2.0}$ & $92.6_{-2.5}^{+2.6}$ & \\
\hline
\end{tabular}

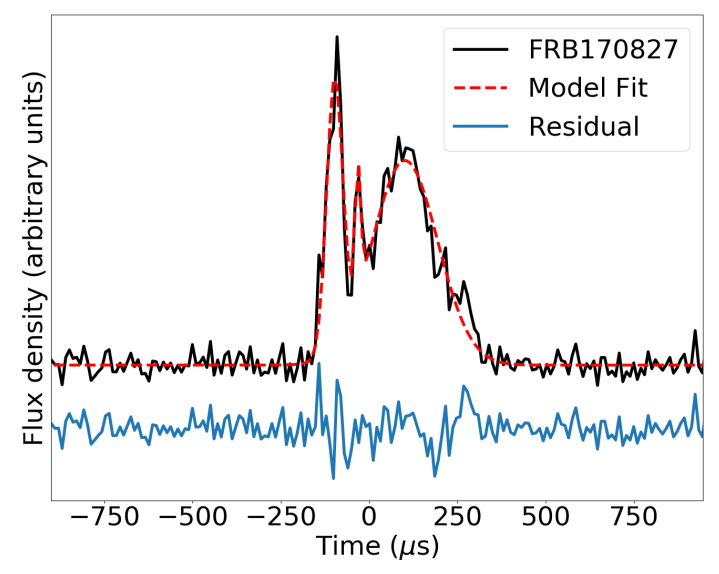

Figure 5. Observed pulse profile (black), model fit (red) and the residual (blue, offset for visibility) for temporal profile of FRB170827. The model consists of 3 Gaussian profiles convolved with a one-sided trailing exponential. The model parameters are listed in Table 2 The modeling yields a scattering of $4.1 \pm 2.7 \mu \mathrm{s}$.

scale of the far screen is:

$$
r_{\mathrm{F}, \mathrm{FAR}}=\sqrt{\frac{D_{\mathrm{eff}}}{k\left(1+z_{l}\right)}},
$$

where $D_{\text {eff }}=D_{\mathrm{L}} D_{\mathrm{LS}} / D_{\mathrm{S}}$ and $z_{l}$ are the effective distance and redshift of the scattering material respectively (Macquart \& Koay 2013).

The condition that the more distant screen is not resolved by the Galactic screen is:

$$
r_{0} \gtrsim r_{\text {ref,ISM. }}
$$

Using Eqs. 6 to 10, Eq. 11 reduces to:

$$
\frac{\Delta \nu_{\mathrm{d}, \mathrm{FAR}} \Delta \nu_{\mathrm{d}, \mathrm{ISM}}}{\nu^{2}} \gtrsim \frac{D_{\mathrm{LS}} D_{\mathrm{ISM}}}{D_{\mathrm{S}} D_{\mathrm{L}}}\left(1+z_{l}\right) .
$$

For our values of $\Delta \nu_{\mathrm{d}, \mathrm{ISM}}=1.5 \mathrm{MHz}, \Delta \nu_{\mathrm{d}, \mathrm{IGM}}=0.1$ $\mathrm{MHz}, D_{\mathrm{ISM}}=1 \mathrm{kpc}$, and $D_{\mathrm{S}}=500 \mathrm{Mpc}$, we get $D_{\mathrm{LS}} \lesssim 60$ Mpc. The second screen could therefore lie in the Intergalactic Medium, but is also consistent with a turbulent screen close to the FRB source in its host galaxy. This is similar to the case of FRB110523 (Masui et al. 2015), who found evidence for a screen in the host galaxy based on the scattering and polarisation properties of the FRB.

\section{MULTIWAVELENGTH FOLLOW-UP}

Follow-up observation of the event were made at the radio wavelengths with UTMOST, ASKAP, and Parkes, and at optical wavelengths with SkyMapper (Keller et al. 2007).

(i) UTMOST: FRB170827 occurred while we were running the telescope in FRB transit mode after one of the telescope arm drives failed over a weekend, so that normal pulsar observing was not possible. As the telescope was positioned at $\delta=-65.5 \mathrm{deg}$, sources cross through the 4 degree primary beam in approximately 40 minutes. This represents 1.5 hours of (serendipitous) follow-up of the event in the following 48 hours, and before other telescopes could be triggered. No candidate bursts were found 60 minutes around the event, nor in the data recorded in the following two days. Around 22 hours of follow-up of the FRB were then performed during the period from August 2017 to February 2018, finding no repeat bursts down to a $\mathrm{S} / \mathrm{N}$ of 9 .

(ii) Parkes: We searched for repeat bursts using the 20 $\mathrm{cm}$ multibeam receiver and the BPSR backend, as part of the SUPERB project (Keane et al. 2018). The observations were taken in the frequency range $1182-1582 \mathrm{MHz}$, with a usable bandwidth of $320 \mathrm{MHz}$, using 1024 channels of width $390 \mathrm{kHz}$ each. Nineteen grid points were searched for 300 seconds each along the localisation arc as provided in Eq. 1 . spaced around 20 arcminutes, starting at UTC 2017-08-30 16:39:29. No bursts were found with $\mathrm{S} / \mathrm{N}>8$.

(iii) ASKAP: 12 hours of follow-up beginning on August 30th, 2017, 22:33:17 UTC, centred at the best-fitting position of FRB170827's field was performed with a single antenna. The observations were taken at a central frequency of $1300 \mathrm{MHz}$ with a bandwidth of $300 \mathrm{MHz}$. No bursts were detected above a $\mathrm{S} / \mathrm{N}$ of 9.5 , corresponding to a limiting fluence of $22 \mathrm{Jy} \mathrm{ms}$, at the $1.26 \mathrm{~ms}$ time resolution.

(iv) SkyMapper: Several nights of imaging data were taken in the week after the event (2017-08-29, 2017-08-30, 2017-09-01). Images were taken in the uvgriz bands (100 s exposures) with photometric depth limits of $u=18.1$, $v=18.5, g=20.5, r=20.3, i=19.5$ and $z=18.7$, at the 95\% upper limit provided by the SkyMapper Transient Survey Pipeline (Scalzo et al. 2017). The follow-up fields were centred around the FRB coordinates, extending north-south to cover the 2 -sigma error regions (i.e. 4.8 degrees). We carried out two different follow-up modes over two nights: the first consists on images centred on the FRB position with multiple visits with slight pointing offsets, and the second takes images of the SkyMapper field covering the FRB localisation and the 2-sigma regions.

Eight galaxies were found in the 6dFGS catalog (Jones et al. 2009) lying along the localisation arc of the FRB, in the redshift range $0.005<z<0.087$. They lie in the magnitude ranges $13.6<r_{F}<16.3 ; 12.8<B_{J}<16.3$, and are mostly disk galaxies. Reference images were taken on 2015-07-06 ( $\sim 2$ years ago) and on 2017-09-02 ( $\sim 4$ days after the first epoch of the FRB followup observations). Only five galaxies are well placed on the CCD images to permit proper processing with our image subtraction procedure using both the newly acquired data and the reference images. No transient or variable candidates were detected in the SkyMapper data along the localisation arc. 


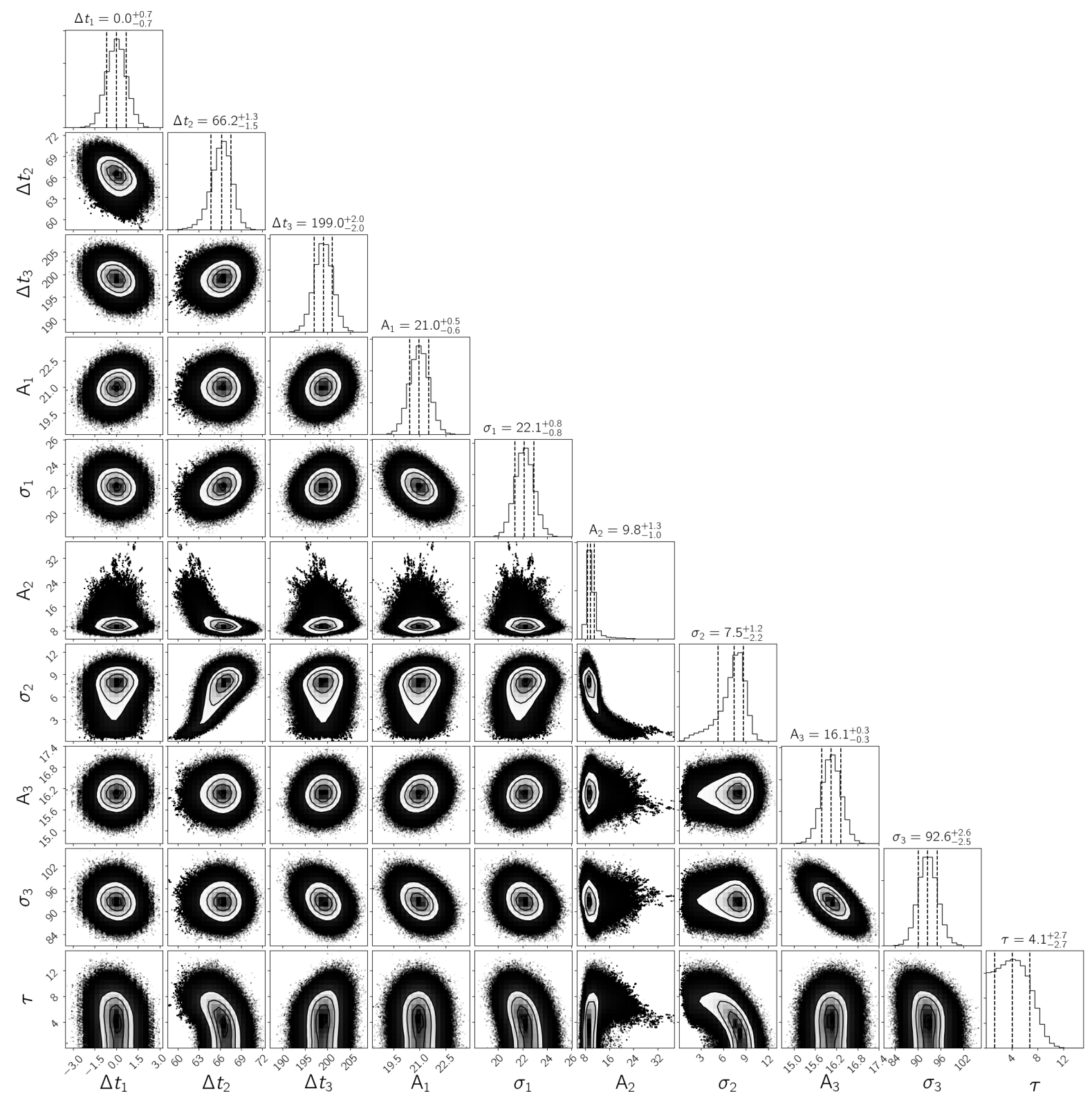

Figure 6. Posterior distributions of the model parameters described in Eq. 5 The dashed lines represent 16, 50 and 84 percentiles in the 1-D histograms.

\section{DISCUSSION AND CONCLUSIONS}

In this paper, we reported a Fast Radio Burst (FRB170827) discovered in near real-time at the Molonglo radio telescope. This demonstrated our ability to trigger voltage capture for a new FRB with a low-latency machine-learning-based discovery system. The discovery allowed, and for the first time, to perform coherent dedispersion of a burst after its blind detection, unveiling temporal structure that would not have been otherwise observed.

The full width of the burst is $400 \mu \mathrm{s}$, one of the narrowest FRBs. It has a DM of $176.80 \pm 0.04 \mathrm{pc} \mathrm{cm}^{-3}$ (after coherent dedispersion), which is the lowest known DM of the FRB population. The Milky Way contribution along the line of sight is $\sim 37 \mathrm{pc} \mathrm{cm}^{-3}(\mathrm{NE} 2001)$ or $\sim 26 \mathrm{pc} \mathrm{cm}^{-3}$ (YMW16), leaving an excess of $\sim 140$ or $150 \mathrm{pc} \mathrm{cm}^{-3}$, and limiting its redshift to $z<0.12$, potentially placing it closer than the repeating FRB (FRB121102, for which the host galaxy is at $z=0.193$ ). It has an observed fluence of $>20$ \pm 7 Jy ms, placing it amongst the brightest FRBs found to date.

The dynamic spectrum of the FRB shows spiky emission features of up to $1 \mathrm{kJy}$ at $10 \mu \mathrm{s}$ and $97.66 \mathrm{kHz}$ resolution, similar to the very bright (50 Jy ms) FRB150807 (Ravi et al. 2016), who reported bright spikes of over $1 \mathrm{kJy}$ at a resolution of $64 \mu \mathrm{s}$ and $390 \mathrm{kHz}$.

The temporal profile of the burst shows three components, the narrowest of which is $\sim 30 \mu \mathrm{s}$. FRB170827 shows spectral modulations on two frequency scales of $\sim 1.5 \mathrm{MHz}$ and $0.1 \mathrm{MHz}$. The latter is based on the scattering of the event of $4.1 \pm 2.7 \mu \mathrm{s}$, obtained via maximum likelihood fit- 
ting of the three burst components. These two scales are clearly visible in the dynamic spectrum of the event which is dominated by a bright region of emission between 841 and $843 \mathrm{MHz}$, but with weaker, patchy emission across the entire band. The present patchy emission is similar to what is seen in the repeating FRB (FRB121102; Michilli et al. 2018) at $4.5 \mathrm{GHz}, \mathrm{FRB} 110523$ (Masui et al. 2015) at 800 $\mathrm{MHz}$ and in FRBs found at ASKAP (Shannon et al. in prep) at $1.4 \mathrm{GHz}$. A decorrelation bandwidth of $1.5 \mathrm{MHz}$ (at $843 \mathrm{MHz}$ ) is expected due to the ISM at the position of FRB170827 $\left((l, b)=\left(303.29^{\circ},-51.58^{\circ}\right)\right)$ using the NE2001 model, which is consistent with the larger of these scales. The $0.1 \mathrm{MHz}$ scale striation is significantly lower than can be accounted for from the ISM, and we speculate it could arise in the host galaxy of the FRB, similarly to the high-RM FRB110523 and the repeating FRB (FRB121102).

Micro-structure visible in the temporal profile of FRB170827 is very similar to that seen in the repeating FRB121102. This and other similarities to the repeater has strongly motivated a follow-up campaign for repeat bursts, currently being conducted at UTMOST at $843 \mathrm{MHz}$ to a fluence limit of approximately $5 \mathrm{Jy} \mathrm{ms}$ and Parkes at $1.4 \mathrm{GHz}$ to a limit of approximately $0.5 \mathrm{Jy} \mathrm{ms}$.

We are currently upgrading the interferometer's second arm (UTMOST-2D project), and our FRB detection and voltage dump system will enable us to perform FRB host galaxy localisation to a few arcsec accuracy from single FRB events by dumping voltages across the array, such as was achieved for FRB170827.

\section{ACKNOWLEDGMENTS}

The Molonglo Observatory is owned and operated by the University of Sydney, with support from the School of Physics and the University. The UTMOST project is also supported by the Swinburne University of Technology. We acknowledge the Australian Research Council grants CE110001020 (CAASTRO) and the Laureate Fellowship FL150100148. We thank Dave Temby, Glen Torr, Glenn Urquhart, Simon Jordan for cheerfully keeping the UTMOST facility performing so smoothly. We thank Kiyoshi Masui for helpful discussions. The Parkes radio telescope is part of the Australia Telescope National Facility, which is funded by the Commonwealth of Australia for operation as a National Facility managed by CSIRO. The ASKAP is part of the Australia Telescope National Facility, which is managed by CSIRO. Operation of ASKAP is funded by the Australian Government with support from the National Collaborative Research Infrastructure Strategy. ASKAP uses the resources of the Pawsey Supercomputing Centre. Establishment of ASKAP, the Murchison Radio-astronomy Observatory and the Pawsey Supercomputing Centre are initiatives of the Australian Government, with support from the Government of Western Australia and the Science and Industry Endowment Fund. We acknowledge the Wajarri Yamatji people as the traditional owners of the Observatory site. ATD is supported by an ARC Future Fellowship grant FT150100415. Parts of this work was performed on the gSTAR national facility at Swinburne University of Technology. SkyMapper is owned and operated by The Australian National University's Research School of Astronomy and As- trophysics. The national facility capability for SkyMapper has been funded through ARC LIEF grant LE130100104 from the Australian Research Council, awarded to the University of Sydney, the Australian National University, Swinburne University of Technology, the University of Queensland, the University of Western Australia, the University of Melbourne, Curtin University of Technology, Monash University and the Australian Astronomical Observatory.

\section{REFERENCES}

Bailes M., et al., 2017, Publ. Astron. Soc. Australia 34, e045

Bandura K., 2014, in 2014 United States National Committee of URSI National Radio Science Meeting (USNC-URSI NRSM). pp 1-1, doi:10.1109/USNC-URSI-NRSM.2014.6928108

Bannister K. W., et al., 2017, ApJ |841, L12

Bassa C. G., et al., 2017, ApJ 843, L8

Bhandari S., et al., 2018, MNRAS 475, 1427

Breiman L., 2001, Machine Learning 45, 5

Burke-Spolaor S., Bannister K. W., 2014, ApJ, 792, 19

Caleb M., et al., 2016, MNRAS, 458, 718

Champion D. J., et al., 2016, MNRAS, 460, L30

Chatterjee S., et al., 2017, Nature 541, 58

Cordes J. M., 1986, ApJ, 311, 183

Cordes J. M., Lazio T. J. W., 2002, ArXiv Astrophysics e-prints,

Cordes J. M., Wasserman I., 2016, MNRAS 457, 232

Cordes J. M., Boriakoff V., Weisberg J. M., 1983, ApJ, 268, 370

Cordes J. M., Bhat N. D. R., Hankins T. H., McLaughlin M. A., Kern J., 2004, ApJ 612, 375

Eftekhari T., Berger E., 2017, ApJ , 849, 162

Falcke H., Rezzolla L., 2014, A\&A 562, A137

Farah W., et al., 2017, The Astronomer's Telegram, 10697

Foreman-Mackey D., 2016, The Journal of Open Source Software. 24

Foreman-Mackey D., Hogg D. W., Lang D., Goodman J., 2013, PASP 125, 306

Inoue S., 2004, MNRAS 348, 999

Jones D. H., et al., 2009, MNRAS 399, 683

Keane E. F., Stappers B. W., Kramer M., Lyne A. G., 2012, MNRAS 425, L71

Keane E. F., et al., 2016, Nature 530, 453

Keane E. F., et al., 2018, MNRAS 473, 116

Keith M. J., et al., 2010, MNRAS, 409, 619

Keller S. C., et al., 2007, Publ. Astron. Soc. Australia 24, 1

Law C. J., et al., 2015, ApJ 807, 16

Lorimer D. R., Kramer M., 2012, Handbook of Pulsar Astronomy

Lorimer D. R., Bailes M., McLaughlin M. A., Narkevic D. J., Crawford F., 2007, Science, 318, 777

Macquart J.-P., Ekers R. D., 2018, MNRAS 474, 1900

Macquart J.-P., Koay J. Y., 2013, ApJ 776, 125

Manchester R. N., Fan G., Lyne A. G., Kaspi V. M., Crawford F., 2006, ApJ, 649, 235

Marcote B., et al., 2017, ApJ 834, L8

Masui K., et al., 2015, Nature, 528, 523

Metzger B. D., Berger E., Margalit B., 2017, ApJ, 841, 14

Michilli D., et al., 2018, Nature 553, 182

Palaniswamy D., Li Y., Zhang B., 2018, ApJ 854, L12

Pen U.-L., Connor L., 2015, ApJ 807, 179

Petroff E., et al., 2015, MNRAS 447, 246

Petroff E., et al., 2016, Publ. Astron. Soc. Australia, 33, e045

Petroff E., et al., 2017, MNRAS 469, 4465

Ravi V., 2017, preprint, (arXiv:1710.08026)

Ravi V., Shannon R. M., Jameson A., 2015, ApJ 799, L5

Ravi V., et al., 2016, Science, 354, 1249

Scalzo R. A., et al., 2017, Publ. Astron. Soc. Australia, 34, e030

Spitler L. G., et al., 2014, ApJ 790, 101 
10 Wael Farah et al.

Spitler L. G., et al., 2016, Nature 531, 202

Tendulkar S. P., et al., 2017, ApJ 834, L7

Totani T., 2013, PASJ 65, L12 